\title{
Impact of decreasing cerebrospinal fluid enterovirus PCR turnaround time on costs and management of children with suspected enterovirus meningitis
}

\author{
Mohammad Alghounaim ${ }^{1,2} \cdot$ Chelsea Caya ${ }^{3} \cdot$ MinGi Cho $^{3} \cdot$ Marc Beltempo ${ }^{3,4} \cdot$ Cedric P. Yansouni ${ }^{2,5}$. \\ Nandini Dendukuri ${ }^{3,6} \cdot$ Jesse Papenburg ${ }^{1,2,3,6,7}$ iD
}

Received: 31 October 2019 / Accepted: 12 December 2019 / Published online: 13 January 2020

(C) Springer-Verlag GmbH Germany, part of Springer Nature 2020

\begin{abstract}
To estimate the impact of implementing in-hospital enterovirus (EV) polymerase chain reaction (PCR) testing of cerebrospinal fluid (CSF) with same-day turn-around-time (TAT) on length-of-stay (LOS), antibiotic use and on cost per patient with suspected EV meningitis, compared with testing at an outside reference laboratory. A model-based analysis using a retrospective cohort of all hospitalized children with CSF EV PCR testing done between November 2013 and 2017. The primary outcome measured was the potential date of discharge if the EV PCR result had been available on the same day. Patients with positive EV PCR were considered for potential earlier discharge once clinically stable with no reason for hospitalization other than intravenous antibiotics. Descriptive statistics and cost-sensitivity analyses were performed. CSF EV PCR testing was done on 153 patients, of which 44 (29\%) had a positive result. Median test TAT was 5.3 days (IQR 3.9-7.6). Median hospital LOS was 5 days (IQR 3-12). Most $(86 \%)$ patients received intravenous antibiotics with mean duration of $5.72 \pm 6.51$ days. No patients with positive EV PCR had a serious bacterial infection. We found that same-day test TAT would reduce LOS and duration of intravenous antibiotics by 0.50 days $(95 \%$ CI $0.33-0.68)$ and 0.67 days (95\%CI $0.42-0.91)$, respectively. Same-day test TAT was associated with a cost reduction of 342.83CAD (95\%CI 178.14-517.00) per patient with suspected EV meningitis. Compared with sending specimens to a reference laboratory, performing CSF EV PCR in-hospital with same-day TAT was associated with decreased LOS, antibiotic therapy, and cost per patient.
\end{abstract}

Keywords Molecular diagnostic techniques - Central nervous system · Economic evaluation · Simulated cohort · Pleocytosis · Infant $\cdot$ Newborn

$\begin{array}{ll}\text { Abbreviation } \\ \text { CAD } & \text { Canadian dollar } \\ \text { CNS } & \text { Central nervous system } \\ \text { CSF } & \text { Cerebrospinal fluid }\end{array}$

Jesse Papenburg

jesse.papenburg@mail.mcgill.ca

1 Division of Pediatric Infectious Diseases, Department of Pediatrics, Montreal Children's Hospital, McGill University Health Centre, Montreal, Quebec, Canada

2 Division of Microbiology, Department of Clinical Laboratory Medicine, McGill University Health Centre, Montreal, Quebec, Canada

3 Centre for Outcomes Research and Evaluation, McGill University Health Centre - Research Institute, Montreal, Quebec, Canada, McGill University, Montreal, Quebec, Canada

$\begin{array}{ll}\text { EV } & \text { Enterovirus } \\ \text { LOS } & \text { Length of stay } \\ \text { MUHC } & \text { McGill University Health Centre } \\ \text { PCR } & \text { Polymerase chain reaction }\end{array}$

4 Division of Neonatology, Department Pediatrics, Montreal Children's Hospital, McGill University Health Centre, Montreal, Quebec, Canada

5 Division of Infectious Diseases, Department of Medicine, McGill University Health Centre, Montreal, Quebec, Canada

6 Department of Epidemiology, Biostatistics and Occupational Health, McGill University, Montreal, Quebec, Canada

7 The Montreal Children's Hospital, E05.1905 - 1001 Décarie Blvd, Montréal, Quebec H4A 3J1, Canada 
SBI Serious bacterial infection

TAT Turn-around-time

\section{Introduction}

Enteroviruses (EVs) are the most common cause of aseptic meningitis, accounting for 48-95\% of all cases for which an etiological agent is identified [1,2]. The clinical course of central nervous system (CNS) EV disease is mostly mild and self-resolving [3, 4]. However, EV infection can be clinically indistinguishable from some serious bacterial infections (SBI), including meningitis. This results in potentially unnecessary hospitalization and empiric intravenous antibiotic therapy [5]. With a positive EV polymerase chain reaction (PCR) result on a cerebrospinal fluid (CSF) sample, clinicians can be highly confident that the illness is not bacterial in origin. Thus, identifying EV in the CSF has been shown to shorten hospital length of stay (LOS) and antibiotic therapy duration $[3,6]$.

PCR is the current "gold-standard" test for EV detection in the CSF and other body fluids [6]. It has sensitivity and specificity approaching $100 \%$ with results available in as short as $\sim 1 \mathrm{~h}$ [5]. At our center, CSF EV PCR testing is sent out to a reference laboratory, with long TATs due to sample transport, batching, and delays in result transmission. This practice also increases the risk of specimen loss or quality degradation, and transcriptional errors. However, there are now commercially available one-step, random-access assays approved by the FDA that can identify EV in the CSF in $\sim 1 \mathrm{~h}$, allowing for same-day results $[7,8]$. We hypothesized that, compared to sending CSF to a reference laboratory for EV PCR, testing inhospital with same-day TAT would be associated with improved patient care and reduced costs per patient with suspected EV meningitis from the healthcare system perspective. Thus, we aimed to compare our current practice (based on send-out testing) with a simulated scenario of CSF EV PCR testing in-house with same-day results. Our primary objective was to estimate the potential impact of same-day CSF EV PCR results on hospital LOS and intravenous antibiotic use. Our secondary objective was to determine the potential economic impact, per tested patient with suspected EV meningitis, of in-hospital testing with same-day TAT.

\section{Methods}

\section{Study design and population}

We performed a single-center model-based analysis of a retrospective cohort at the Montreal Children's Hospital of the McGill University Health Centre (MUHC), Montreal, Quebec, Canada, a 149-bed tertiary/quaternary pediatric hospital with $>5000$ admissions annually. We included all admitted patients $<18$ years old with suspected EV meningitis, defined as patients who had EV PCR performed on a CSF specimen, from November 1, 2013 to November 30, 2017. We excluded patients that were not admitted.

\section{Data collection}

We extracted data pertaining to the hospitalization from medical records, including patient demographics, clinical characteristics, intravenous antibiotic and/or antiviral use, microbiological testing, and other laboratory values (CSF cell count and biochemistry). CSF pleocytosis was defined according to patient age: $>15$ cells $/ \mu \mathrm{L}$ for subjects $\leq 28$ days of age, $>$ 9 cells $/ \mu \mathrm{L}$ in subjects $29-60$ days old, and $>6$ cells $/ \mu \mathrm{L}$ in subjects $>60$ days old [9-11].

\section{Microbiological testing}

Blood and CSF specimens for EV PCR testing were forwarded to the reference lab (CHU Sainte-Justine, Montreal, Quebec, Canada) every working day at 8 a.m. Specimens received after 8 a.m. on Friday through Sunday were frozen at $-70{ }^{\circ} \mathrm{C}$ and sent on the following Monday. The reference center test was a laboratory-developed real-time PCR assay performed during regular working days. Typically, results were transmitted by fax at the end of the day. Results were then entered into the MUHC laboratory information system. Test TAT was defined as the time between specimen collection and notification of final result through the hospital's electronic system. Multiplex PCR testing (including EV) on respiratory specimens and stool viral cultures were done as previously described $[12,13]$.

\section{Estimation of the clinical impact of EV CSF PCR same-day TAT by a simulated cohort}

For the simulated cohort of children with same-day results, we estimated a potential earlier date of discharge or discontinuation of intravenous antibiotics was determined by reviewing daily notes in patients' medical records. A negative CSF EV PCR was assumed not to change LOS or antibiotic duration. For cases with a positive CSF EV PCR, the simulated discharge date was judged to be the earliest hospitalization day that fulfilled the following criteria: (1) clinical stability (no need for supplemental oxygen, intravenous fluids or continuous monitoring of vital signs; no uncontrolled seizures or altered level of consciousness), (2) no need for intravenous antibiotics for reasons other than the clinical syndrome for which they tested positive for EV, and (3) no need for other in-patient treatments or investigations. For admitted neonates $(\leq 28$ days of age), along with the aforementioned criteria, estimated antibiotic stop date and discharge date were always estimated to 
be at minimum $24 \mathrm{~h}$ after collection of appropriate cultures [14-16].

\section{Economic evaluation}

We assessed inpatient medical costs from the perspective of the provincial healthcare system consisting of costs incurred to the single public payer. The time horizon of the analysis was restricted to the episode of acute care of each patient; therefore, costs were not discounted. This included the hospitalization associated with the CSF EV PCR testing and subsequent readmission or Pediatric Day Center visits directly related to the admission. Only direct healthcare costs were included in the analysis. Indirect costs were not included because data for potential productivity losses of parents were not available. All costs were measured and reported in 2018 Canadian dollars (CAD).

For the economic analysis, cost information for the various elements of clinical care were obtained from the following sources (Table 1). Per diem costs of hospitalization (general ward, pediatric intensive care unit, and neonatal intensive care unit) and Pediatric Day Center visits were

Table 1 Summary of included cost items and unit prices

\begin{tabular}{ll}
\hline & Cost (CAD) \\
\hline CSF EV PCR test (per test) & \\
Current test at reference laboratory & 34 \\
Estimated cost of in-hospital test & 175 \\
Hospitalization (per day) & \\
General pediatric ward & 613 \\
Pediatric intensive care & 1465.5 \\
Neonatal intensive care & 1465.5 \\
Medical day hospital & 267.6 \\
Antimicrobials (per gram) & \\
Acyclovir & 10.95 \\
Ampicillin & 3.80 \\
Cefotaxime & 4.01 \\
Ceftriaxone & 1.08 \\
Meropenem & 7.30 \\
Tobramycin & 520.5 \\
Vancomycin & 3.06 \\
Physician fee for service (per visit) & \\
Pediatric team primary visit & $143.20-337.03$ \\
Pediatric team follow-up visits & $25.35-125.00$ \\
Subspecialist primary visit (consult) & $157.00-434.5$ \\
Subspecialist follow-up visits & $35.5-69.85$ \\
\hline
\end{tabular}

$C A D$ Canadian dollars, $C S F$ cerebrospinal fluid, $E V$ enterovirus, $P C R$ polymerase chain reaction

${ }^{a}$ The exact fee for physician billing per service varies depending on the day of the week, inpatient vs medical day hospital, and the subspecialty of the physician obtained from the MUHC Finance Department. Costs of intravenous medications were obtained from MUHC Pharmacy. Physician billing costs were based on the Régie de l'Assurance Maladie du Québec billing handbook, and vary according to weekday/weekend visit, the specialty of the consultant, the care unit the patient was admitted to, and the age of the patient [17]. To estimate physician billing costs, we assumed that the treating physician billed for two visits per day, which corresponds to morning and afternoon clinical rounds. We assumed that consultant services would charge for a consultation on the first visit and follow-up visits for each subsequent documented patient encounter, to a maximum of two visits per day. Regarding EV CSF PCR testing, the Quebec Ministry of Health assigns a value of $34 \mathrm{CAD}$ per sample to the assay performed at the reference laboratory and we estimated local CSF EV PCR testing would cost 175 CAD per sample [18]. In our center, current laboratory facilities and infrastructure could allow the implementation of CSF EV PCR. Hence, capital costs for implementing in-hospital testing (i.e., acquisition of new instruments/platforms) were not included.

\section{Data analysis}

Data were presented using descriptive statistics. Means were compared using the paired Student $t$ test and mean differences calculated with corresponding $95 \%$ confidence intervals (95\% CI).

We compared the mean LOS, antimicrobial duration (antibacterials and antivirals), and costs per patient with suspected EV meningitis in the observed cohort of children with send-out testing to those of the simulated cohort of children with same-day results. The difference in average costs of care per patient between the two scenarios (i.e., observed reference laboratory testing vs. simulated inhospital testing) was used to estimate the economic impact of same-day EV PCR TAT. Because the economic effects of a diagnostic strategy may depend on disease characteristics, pretest probability (i.e., disease prevalence in population tested), and test cost, sensitivity analyses were performed to assess the impact of changes in key variables used in the analysis. Pretest probability was varied from $15-45 \%$, in-hospital test cost from $34 \$-300 \$$, test TAT lengthened to $48 \mathrm{~h}$, and testing restricted to peak EV season (May to October or July to October) or to patients with pleocytosis or young infants $<60$ days of age. Finally, we performed additional economic sensitivity analyses using the lower bounds of the $95 \%$ CIs of the estimated mean differences in LOS and duration of antimicrobial use, thereby assessing if findings of cost reductions would be robust to the most conservative assessment of the clinical benefits of in-hospital testing. 


\section{Ethics}

This study was approved by the McGill University Health Centre research ethics board (2018-4190).

\section{Results}

During the study period, we identified 155 patients in whom a CSF specimen was tested for EV by PCR. Of those, two were not admitted and therefore excluded. Forty-four of 153 patients $(29 \%)$ had a positive EV CSF PCR result. The majority occurred between July and October (Fig. 1). Mean age was 2.80 years, with approximately half under 60 days of age. Median LOS was 5 days (IQR 3-12). Most patients (86\%) received intravenous antibiotics and $39 \%$ received intravenous acyclovir, with mean durations of 5.72 and 4.45 days, respectively (Table 2 ).

CSF pleocytosis was detected in $90.9 \%$ of CSF EVpositive specimens and in $54 \%$ of CSF EV-negative samples. Three of the 44 EV-positive patients had a normal CSF leukocyte count $(0,2$, and 3 cells/ $\mu \mathrm{L})$. Forty-seven $(30.7 \%)$ patients had EV identified in a non-CSF specimen (Table 2). Among patients who tested positive by CSF EV PCR, 43 $(98 \%)$ had concomitant testing for respiratory viruses (including EV) from an upper respiratory tract specimen. Of those, $58 \%$ tested positive for EV. Stool viral culture was performed in only 16 EV CSF PCR-positive patients (36\%) with nine positive results for $\mathrm{EV}(56 \%)$. No patient with positive $\mathrm{EV}$ CSF PCR had a bacterial pathogen identified in CSF, blood or urine.
Mean TAT for EV CSF PCR was 6.26 days. Among EVpositive patients, $38(86.4 \%)$ met criteria for potential early discharge, and $40(90.9 \%)$ for shorter antimicrobial duration in the simulated cohort. Overall, same-day TAT would decrease mean LOS by 0.50 days (95\% CI $0.33-0.68$ ) and intravenous antimicrobial duration by 0.67 days $(95 \% \mathrm{CI}$ 0.42-0.91). Restricting analyses to EV CSF PCR+ patients, estimated reductions in LOS, and antimicrobial duration were 1.75 days (95\% CI 1.31-2.19) and 2.32 days (95\% CI 1.68-2.96), respectively (Table 3 ).

In economic analysis (Table 4), same-day TAT for EV CSF PCR would save 342.83 CAD (95\%CI, 178.14-517.00) per patient tested for suspected EV infection (13,113.42 CAD annually). In sensitivity analyses, savings varied between 483.83 CAD per patient to 217.83 CAD per patient when the cost per test ranged between $34 \mathrm{CAD}$ and $300 \mathrm{CAD}$, respectively, assuming test performance was the same between different assays (Fig. 2). Disease pretest probability also affected economic impact; the break-even point was observed with disease pretest probability between 8.0 and $8.5 \%$ (Fig. 3). Similarly, limiting testing to high EV season (July to October) was associated with increased savings (549.06 CAD per patient). Also, savings increased if testing was limited to patients with CSF pleocytosis (555.44 CAD per patient) or to infants $<60$ days of age (571.00 CAD per patient). Savings decreased to $90.73 \mathrm{CAD}$ per patient if test TAT was extended to $48 \mathrm{~h}$. Finally, using the lower bounds of the $95 \%$ CIs for the mean differences in LOS and antimicrobial duration to estimate the minimal potential cost reductions that could be achieved, mean cost savings would be 178.14 CAD per patient and there would be potential cost savings in all variables tested in sensitivity analyses (Fig. 4).
Fig. 1 The histogram illustrates the total number of enterovirus CSF PCR tests performed as well as the proportion among which were positive for enterovirus by calendar month from Nov. 2013 to Nov. 2017. CSF, cerebrospinal fluid; PCR, polymerase chain reaction

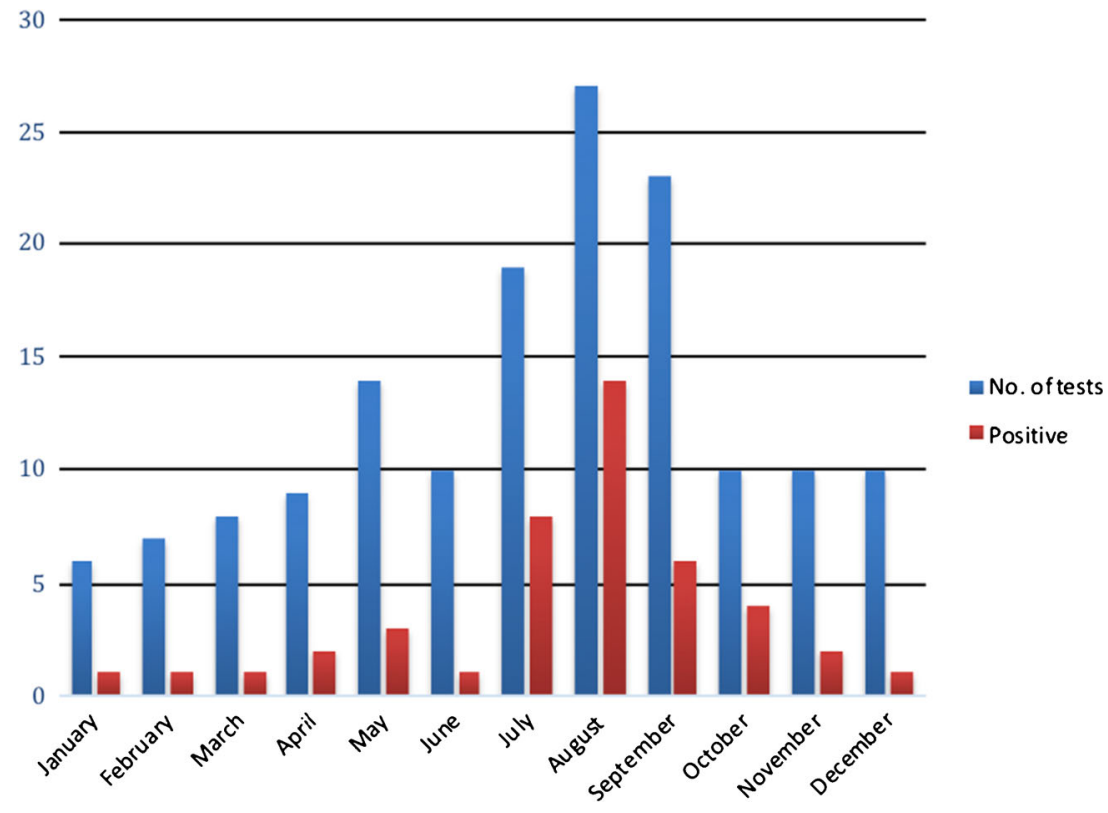


Table 2 Demographic, clinical and microbiological information

\begin{tabular}{|c|c|c|c|}
\hline & $\begin{array}{l}\text { CSF enterovirus }+ \\
n=44(\%)\end{array}$ & $\begin{array}{l}\text { CSF } \\
\text { Enterovirus - } \\
n=109(\%)\end{array}$ & $\begin{array}{l}\text { TOTAL } \\
n=153(\%)\end{array}$ \\
\hline Age at time of CSF collection, years (mean, SD) & $2.15 \pm 4.22$ & $3.06 \pm 4.40$ & $2.80 \pm 4.36$ \\
\hline Infants $<60$ days old $(n, \%)$ & $30(68.2)$ & $41(37.6)$ & $71(46.4)$ \\
\hline Male $(n, \%)$ & $28(63.6)$ & $59(54.1)$ & $87(56.8)$ \\
\hline Hospital length of stay (median, IQR) & $\begin{array}{l}3.00 \\
(3.00-4.00)\end{array}$ & $\begin{array}{l}7.00 \\
(4.00-17.00)\end{array}$ & $\begin{array}{l}5.00 \\
(3.00-12.00)\end{array}$ \\
\hline \multicolumn{4}{|l|}{ Primary discharge diagnosis } \\
\hline Bacterial meningitis & $2(4.5)$ & $15(13.7)$ & $17(11.1)$ \\
\hline Viral meningitis & $38(86.3)$ & $22(20.1)$ & $60(39.2)$ \\
\hline Infectious encephalitis & 0 & $6(5.5)$ & $6(3.9)$ \\
\hline Post infectious & 0 & $4(3.6)$ & $4(2.6)$ \\
\hline Noninfectious & 0 & $8(7.3)$ & $8(5.2)$ \\
\hline Non-CNS infection & $3(6.8)$ & $17(15.5)$ & $20(13.0)$ \\
\hline Genetic/inborn error of metabolism & 0 & $4(3.6)$ & $4(2.6)$ \\
\hline CNS disease (non-infectious) & $1(2.2)$ & $6(5.5)$ & $7(4.5)$ \\
\hline Seizure & 0 & $11(10.0)$ & $11(7.1)$ \\
\hline Suspected sepsis & 0 & $2(1.8)$ & $2(1.3)$ \\
\hline Other & 0 & $13(11.9)$ & $13(8.4)$ \\
\hline Other CNS infection & 0 & $4(3.6)$ & $9(2.6)$ \\
\hline Intravenous antimicrobials administered $(n, \%)$ & $42(95.4)$ & $90(82.5)$ & $132(86.2)$ \\
\hline LP prior to administration of intravenous antimicrobials $(\mathrm{n}, \%)(n=132)$ & $36(85.7)$ & $46(51.1)$ & $82(62.1)$ \\
\hline Duration of intravenous antimicrobials & $3.83 \pm 2.17$ & $6.60 \pm 7.60$ & $5.72 \pm 6.51$ \\
\hline Mean, SD & 3.00 & 4.00 & 3.00 \\
\hline Median, IQR & $(3.00-4.00)$ & $(3.00-6.00)$ & $(3.00-5.00)$ \\
\hline Intravenous antivirals administered $(n, \%)$ & $11(25.0)$ & $49(44.9)$ & $60(39.2)$ \\
\hline Duration of intravenous antivirals & $2.72 \pm 2.53$ & $4.83 \pm 3.47$ & $4.45 \pm 3.40$ \\
\hline Mean, SD & 2.00 & 4.00 & 4.00 \\
\hline Median, IQR & $(1.00-2.50)$ & $(3.00-6.00)$ & $(2.00-6.00)$ \\
\hline \multicolumn{4}{|l|}{ CSF parameters } \\
\hline WBC (median, IQR) & $\begin{array}{l}178.0 \\
(47.5-511.5)\end{array}$ & $\begin{array}{l}16.0 \\
(2.0-139.0)\end{array}$ & $\begin{array}{l}34.5 \\
(4.0-212.0)\end{array}$ \\
\hline Pleocytosis $(n, \%)$ & $40(90.9)$ & $59(54.1)$ & $99(64.7)$ \\
\hline RBC (cells $/ \mu \mathrm{L} ;$ median, IQR) & $\begin{array}{l}25.0 \\
(1.0-452.2)\end{array}$ & $\begin{array}{l}9.0 \\
(1.0-160.8)\end{array}$ & $\begin{array}{l}10.5 \\
(1.0-231.8)\end{array}$ \\
\hline Protein (cells $/ \mu \mathrm{L} ;$ median, IQR) & $\begin{array}{l}0.79 \\
(0.43-1.09)\end{array}$ & $\begin{array}{l}0.60 \\
(0.30-1.14)\end{array}$ & $\begin{array}{l}0.67 \\
(0.35-1.12)\end{array}$ \\
\hline CSF enterovirus TAT in days (collection to result) & $6.50 \pm 3.31$ & $6.16 \pm 5.21$ & $6.26 \pm 4.74$ \\
\hline Mean, SD & 5.92 & 4.95 & 5.29 \\
\hline Median, IQR & $(4.41-8.11)$ & $(3.76-6.89)$ & $(3.90-7.59)$ \\
\hline CSF enterovirus TAT in days (in-lab time to result) & $4.78 \pm 2.59$ & $4.43 \pm 4.56$ & $4.53 \pm 4.08$ \\
\hline Mean, SD & 4.54 & 3.77 & 4.00 \\
\hline Median, IQR & $(2.81-6.09)$ & $(2.20-5.81)$ & $(2.23-5.93)$ \\
\hline \multicolumn{4}{|l|}{ CSF HSV PCR } \\
\hline Positive & 0 & $2(1.8)$ & $2(1.3)$ \\
\hline Negative & $13(29.5)$ & $56(51.3)$ & $69(45.0)$ \\
\hline Not done & $31(70.4)$ & $51(46.7)$ & $82(53.5)$ \\
\hline \multicolumn{4}{|l|}{ CSF viral culture } \\
\hline Enterovirus $^{\mathrm{a}}$ & $4(9.0)$ & 0 & $4(2.6)$ \\
\hline Virus other than enterovirus & 0 & 0 & 0 \\
\hline
\end{tabular}


Table 2 (continued)

\begin{tabular}{|c|c|c|c|}
\hline & $\begin{array}{l}\text { CSF enterovirus }+ \\
n=44(\%)\end{array}$ & $\begin{array}{l}\text { CSF } \\
\text { Enterovirus - } \\
n=109(\%)\end{array}$ & $\begin{array}{l}\text { TOTAL } \\
n=153(\%)\end{array}$ \\
\hline Negative & $1(2.2)$ & $36(33.0)$ & $37(24.1)$ \\
\hline Not done & $39(88.6)$ & $73(66.9)$ & $112(73.2)$ \\
\hline \multicolumn{4}{|l|}{ Stool viral culture } \\
\hline Enterovirus & $9(20.4)$ & $8(7.3)$ & $17(11.1)$ \\
\hline Virus other than enterovirus ${ }^{\mathrm{b}}$ & 0 & $1(0.9)$ & $1(0.6)$ \\
\hline Negative & $7(15.9)$ & $33(30.2)$ & $40(26.1)$ \\
\hline Not done & $28(63.6)$ & $67(61.4)$ & $95(62.0)$ \\
\hline \multicolumn{4}{|l|}{ Blood enterovirus PCR } \\
\hline Positive & $1(2.2)$ & $1(0.9)$ & $2(1.3)$ \\
\hline Negative & $2(4.5)$ & $6(5.5)$ & $8(5.2)$ \\
\hline Not done & $41(93.1)$ & $102(93.5)$ & $143(93.4)$ \\
\hline \multicolumn{4}{|l|}{ Respiratory virus $\mathrm{PCR}^{\mathrm{c}}$} \\
\hline Enterovirus only & $25(56.8)$ & $11(10.0)$ & $36(23.5)$ \\
\hline Enterovirus/rhinovirus & $3(6.8)$ & $6(5.5)$ & $9(5.8)$ \\
\hline Rhinovirus & $2(4.5)$ & $7(6.4)$ & $9(5.8)$ \\
\hline Adenovirus & 0 & $5(4.5)$ & $5(3.2)$ \\
\hline Other respiratory viruses & $1(2.2)$ & $10(9.1)$ & $11(7.1)$ \\
\hline Negative & $12(27.2)$ & $59(54.1)$ & $71(46.4)$ \\
\hline Not done & $1(2.2)$ & $16(14.6)$ & $17(11.1)$ \\
\hline \multicolumn{4}{|c|}{ Significant positive bacterial culture } \\
\hline CSF culture & 0 & $2(1.8)$ & $2(1.3)$ \\
\hline Blood culture & 0 & $5(4.5)$ & $5(3.2)$ \\
\hline Urine culture & 0 & $4(3.6)$ & $4(2.6)$ \\
\hline \multicolumn{4}{|c|}{ Non-enterovirus pathogen identified by $\mathrm{PCR}^{\mathrm{d}}$} \\
\hline $\mathrm{CSF}(n=71)$ & 0 & $2(2.8)$ & $2(2.8)$ \\
\hline Blood $(n=10)$ & 0 & $1(10.0)$ & $1(10.0)$ \\
\hline
\end{tabular}

Parechovirus testing only available as of April 2017

$C N S$ central nervous system, $C S F$ cerebrospinal fluid, $I Q R$ interquartile range, $H S V$ herpes simplex virus, $L P$ lumbar puncture, $P C R$ polymerase chain reaction, $R B C$ red blood cell count, $S D$ standard deviation, $T A T$ turn-around-time, $W B C$ white blood cell count

${ }^{a} \mathrm{CSF}$ viral culture isolates were identified as coxsakievirus $(n=1)$ and echovirus $(n=3)$

${ }^{\mathrm{b}}$ Stool culture, other virus identified was adenovirus $(n=1)$

${ }^{\mathrm{c}} 5$ patients had more than 1 virus identified. Other respiratory viruses include: parainfluenviruses $1-3(n=5)$; influenza A $(n=2)$; influenza B $(n=1)$; human metapneumovirus $(n=2)$; coronaviruses $(n=1)$; RSV $(n=2)$

${ }^{\mathrm{d}}$ Varicella-zoster virus and parechovirus. Pathogen identified in blood was $N$. meningitides

\section{Discussion}

Implementation of CSF EV PCR testing has previously been shown to reduce healthcare resource utilization by avoiding unnecessary hospitalization and antimicrobial therapy [5, 19-21]. At our institution, the test is available to clinicians, but specimens are sent to a reference laboratory, with associated delays in result reporting. To our knowledge, no study has yet modeled the clinical and economic impact of performing EV PCR locally compared to sending specimens to a reference laboratory. In our simulated cohort, we found that implementing an in-hospital PCR with same-day TAT would reduce hospital LOS and intravenous antibiotic therapy, thereby reducing direct healthcare costs by $342.83 \mathrm{CAD}$ per patient tested.

Consistent with previous literature demonstrating that the greatest impact of CSF EV PCR testing is among patients with a positive result, we assumed that only a positive test result, indicating an etiology that does not require antimicrobial therapy, would affect patient management. Aronson et al. found that infants $\leq 60$ days of age with positive EV PCR had onethird shorter LOS compared to infants with a negative test result. However, a reduction of LOS for the entire population tested was not demonstrated [20]. We estimated that both the 
Table 3 Estimated clinical impact of potential same-day CSF enterovirus PCR results compared to send out to reference laboratory in pediatric patients with suspected meningitis/ encephalitis
Mean difference between observed (send-out) and hypothetical (in-hospital) testing scenarios $(95 \% \mathrm{CI})$

$\begin{array}{ll}\text { All patients }(n=153) & \\ \text { Length of hospital stay (days) } & 0.50 \\ & (0.33-0.68) \\ \text { Duration of intravenous antimicrobial prescription } & 0.67 \\ \quad \text { (days) } & (0.42-0.91) \\ \text { Enterovirus CSF PCR positive subgroup }(n=44) & \\ \quad \text { Length of hospital stay (days) } & 1.75 \\ \quad & (1.31-2.19) \\ \quad \text { Duration of intravenous antimicrobial prescription } & 2.32 \\ \quad \text { (days) } & (1.68-2.96)\end{array}$

$C A D$ Canadian dollars, $C I$ confidence interval, $C S F$ cerebrospinal fluid, $P C R$ polymerase chain reaction

overall population tested and those with a positive EV PCR would have significant reductions in hospital LOS of 0.50 days (95\% CI $0.33-0.68$ ) and 1.75 days (95\% CI 1.31-2.19), respectively.

The economic effect of this diagnostic strategy is positively associated with disease prevalence in the population being tested. The EV positivity rate in our study was high (29\%) and may not be sustained and/or expected in other settings. However, identification of subgroups with highest pretest probability of having EV meningitis can increase the clinical impact of testing and associated cost reductions. One approach could be to limit EV PCR testing to patients with CSF pleocytosis. However, data on the exact frequency of positive EV PCR in patients without pleocytosis and on the effect of testing on LOS in this subgroup are lacking $[3,5]$. In our study, $6.8 \%$ of CSF EV PCR-positive patients did not have CSF pleocytosis, lower than the proportions described in other studies $(12-18 \%)[5,6,20]$. We also estimated greater cost savings among infants $\leq 60$ days of age. Although inhouse testing still demonstrated cost reductions in our simulated cohort when test TAT was extended to $48 \mathrm{~h}$, it was $\sim 75 \%$ less than the base case scenario of same-day TAT. Therefore,

Table 4 Estimated yearly cost differences between the simulated cohort with same-day CSF enterovirus PCR results compared to the observed cohort with send-out to reference laboratory in pediatric patients with suspected enterovirus meningitis, overall and by cost item group

\begin{tabular}{ll}
\hline & Annual cost savings (CAD) \\
\hline In-hospital CSF Enterovirus PCR testing & -5393.25 \\
Hospitalization and medical-day visits & $+13,188.01$ \\
Intravenous antimicrobial treatment & +222.55 \\
Physician fee for services & +5096.11 \\
Overall cost savings & $+13,113.42$ \\
\hline
\end{tabular}

$C A D$ Canadian dollars, $C S F$ cerebrospinal fluid, $P C R$ polymerase chain reaction
EV CSF testing using random access PCR assays that do not require batching may have higher potential for cost reduction. However, other factors (e.g., batch size, cost per run and technologist time) also need to be considered.

The risk of an SBI is very low in patients with positive EV CSR PCR. In a systematic review of studies of infants < 90 days old. Wallace et al. reported SBI proportions between 1.6 and $6.7 \%$ among young infants with positive EV CSF PCR testing. Urinary tract infection was the most common SBI. Bacteremia occurred in less than $1 \%$ and none had bacterial meningitis $[3,20]$. In a recent large cohort study, Aronson et al. found that the prevalence of any SBI among 945 infants $\leq 60$ days old with positive EV CSF testing was $0.8 \%$, with bacteremia accounting for half of all SBIs and no observed cases of bacterial meningitis [20]. Despite these findings, Basmaci et al. raised concerns for potentially missing bacterial CSF coinfection. They described two cases with bacterial co-infection: one presented with partially treated meningitis, and another in an immunocompromised patient [22]. In our study, no patients with positive EV PCR had an SBI. Importantly, $86 \%$ of EV-positive patients had their lumbar puncture performed before administration of antibiotics. Hence, we believe that clinically stable immunocompetent patients with positive EV CSF PCR with no other reason for admission or findings suggestive of bacterial infection can be safely managed through outpatient care.

Positive EV CSF PCR results have previously been associated with shorter courses of intravenous antibiotics [4-6]. In our simulated cohort, implementing in-hospital testing reduced intravenous antibiotic use on average by 2.32 days (95\% CI 1.68-2.96) and 0.67 (95\% CI 0.42-0.91) for CSF EV-positive patients and the entire cohort, respectively. Due to their low purchasing costs, this reduction in intravenous antibiotic use accounted for only $1.2 \%$ of total estimated cost reduction. However, safely reducing unnecessary antibiotic prescribing and its associated risks of bacterial resistance 


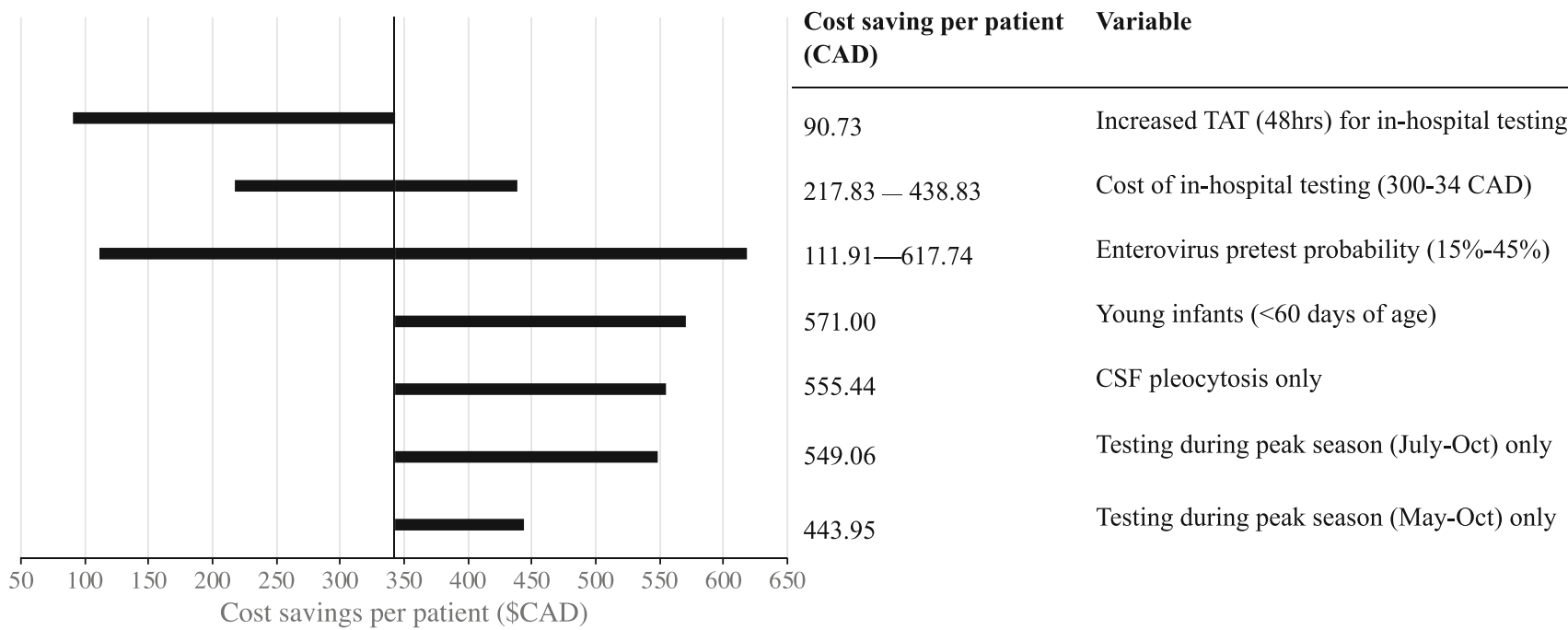

Fig. 2 The tornado diagram indicates the impact on the incremental costs per patient when key variables are varied. All costs in 2018 Canadian dollars. CAD, Canadian dollars; CSF, cerebrospinal fluid; PCR, polymerase chain reaction; TAT, turn-around-time

and adverse drug effects represents an important quality of care improvement.

Enterovirus can be shed in respiratory secretions and stool for weeks, and identification of EV from these sites may suggest that EV is the cause of the meningitis syndrome under investigation, but is not confirmatory. Testing non-CSF specimens can be particularly useful when EV CSF PCR is not available or the testing process is associated with long TAT. In our study, only $57 \%$ of patients with positive EV CSF PCR had a positive result in a respiratory specimen, compared to $10 \%$ in EV CSF
PCR-negative patients. Similarly, in EV CSF PCRpositive patients, nine of $16(56 \%)$ of stool specimens were positive. One can conclude that isolation of EV in non-CSF specimens may increase the likelihood of EV meningitis in patients with pleocytosis. Larger studies, however, are needed to better evaluate the predictive value of detecting EV nucleic acid in non-CSF samples. Of note, some commercially available multiplex PCR assays for respiratory viruses cannot differentiate between rhinovirus and enterovirus due to the genetic similarity of these picornaviruses, which may limit the utility of a positive result from a

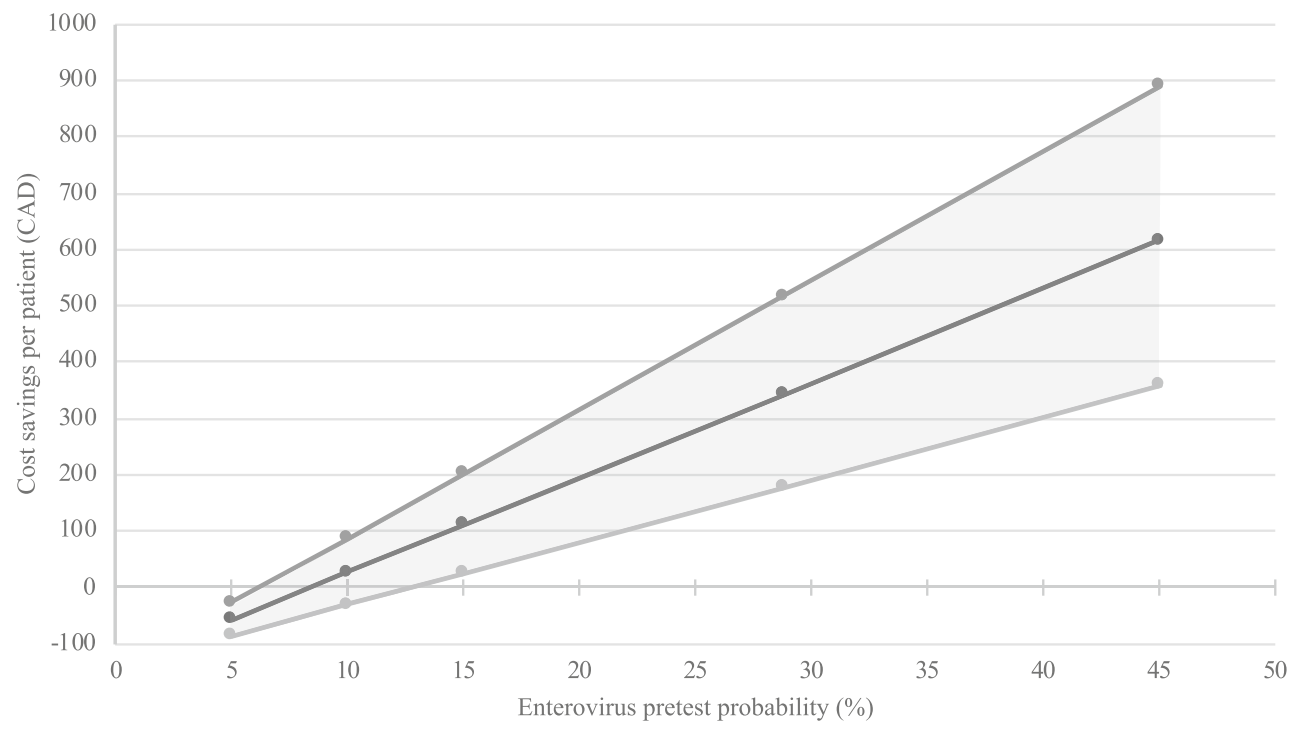

Fig. 3 Potential cost saving per patient for same-day test TAT across a range of enterovirus pretest probabilities. Using the upper and lower bounds of the $95 \%$ CIs for the estimated mean differences in LOS $(0.50 ; 95 \%$ CI $0.33-0.68)$ and duration of antimicrobial use $(0.67 ; 95 \%$ CI 0.42-0.91), we estimated the potential cost savings per patient under the least conservative scenario (i.e., upper bounds of $95 \%$ CI used 0.68 for LOS and 0.91 for duration of antimicrobial use; represented by the top-most line), and the most conservative scenario (i.e., lower bounds of 95\% CI used 0.33 for LOS and 0.42 for duration of antimicrobial use; represented by the bottom-most line) relative to the base-case scenario (i.e., point estimates for LOS (0.50) and duration of antimicrobial use (0.67); represented by the middle line) across a range of enterovirus pretest probabilities. CAD, Canadian dollars; CI, confidence interval; LOS, length of stay; TAT, turn-around-time 


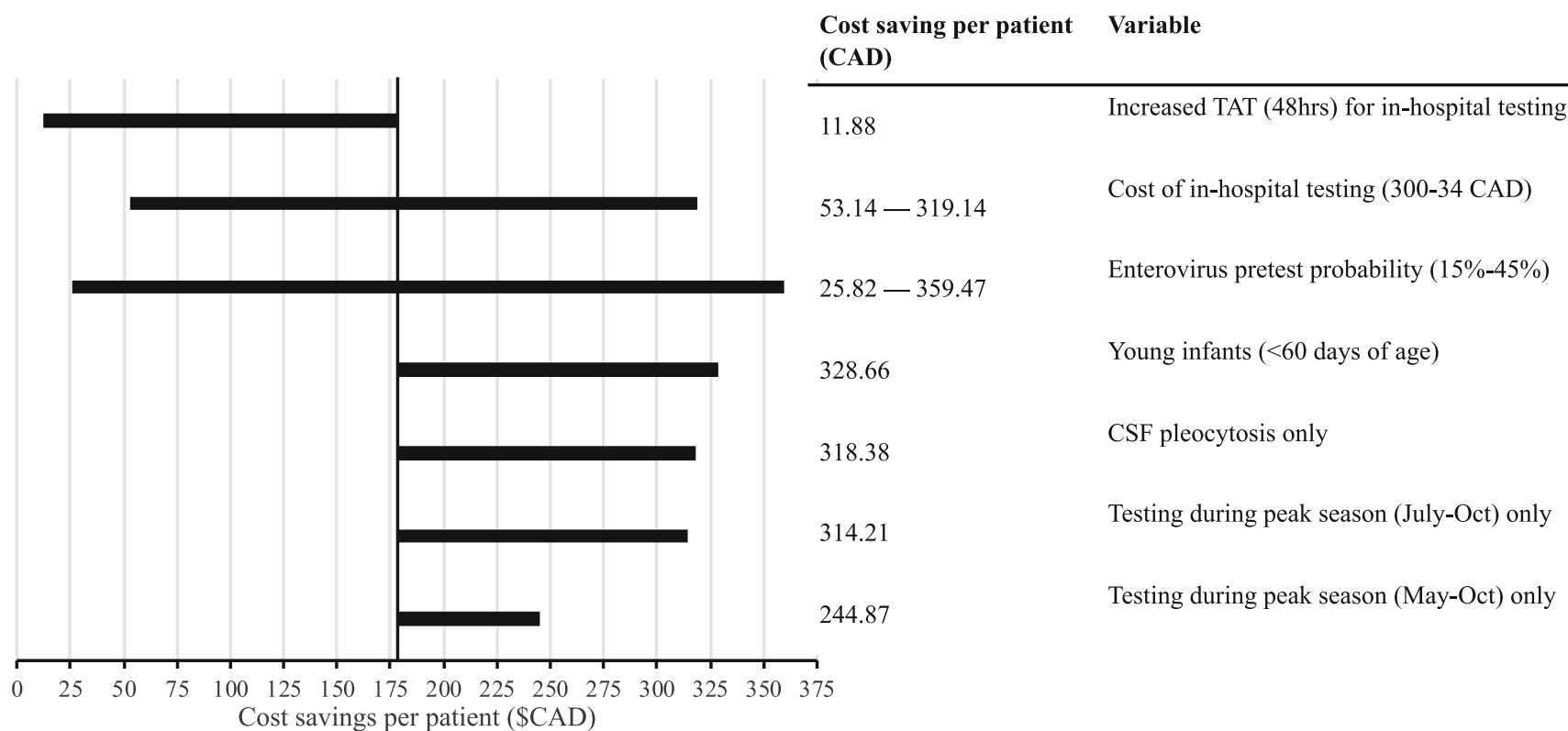

Fig. 4 Sensitivity analyses of the economic impact of same-day EV PCR testing of CSF using the lower range of the $95 \%$ CIs of the estimated mean differences in LOS and duration of antimicrobial use as the average cost reduction per patient with suspected EV meningitis. CAD, Canadian dollars; CSF, cerebrospinal fluid; PCR, polymerase chain reaction; TAT, turn-around-time nasopharyngeal specimen [23]. The laboratory-developed multiplex PCR assay used for respiratory virus testing in our institution discriminates between rhinovirus and enterovirus [13].

This study has several limitations. It was conducted at a single center in a public healthcare system, and results may not be generalizable to other settings. It is also limited by its retrospective nature; estimations of potential earlier discharge dates or earlier cessation of intravenous antimicrobials in our simulated cohort were dependent on the accuracy of documentation in patient charts. Also, in our base-case analysis, we may have underestimated the true cost savings by using the estimated cost of one of the most expensive commercial EV PCR assays approved in Canada for use on CSF specimens. Furthermore, the potential impact on other ancillary testing (e.g., brain imaging) was not assessed, making the study estimates of potential cost reductions more conservative. However, we have addressed uncertainty regarding economic analyses by performing several sensitivity analyses in which we varied the values of key variables associated with costs. We found that in all scenarios, in-hospital testing would be cost saving. We were also limited by not being able to reliably estimate capital costs of acquiring an instrument for in-hospital CSF EV PCR with same-day TAT, for laboratories that did not already have such a platform. This was partly because of the wide variability of costs associated with acquiring new equipment, due to the many different testing platforms available for commercial and laboratory-developed assays. Moreover, instrument acquisition costs, if any, would need to be spread across many different potential assays to be used on the new platform, in addition to EV CSF PCR. Our study could also not assess the possible impact of multiplex testing for other viral targets in the CSF, such as HSV. Finally, patients with suspected aseptic meningitis and/or meningoencephalitis were not systematically tested by EV CSF PCR at our hospital during the study period. Although it would be difficult to predict physicians' test ordering behavior, one could reasonably expect increased test utilization once an assay with a more useful TAT becomes available. Hence, guidance regarding indications for testing and interpretation of results would be needed to optimize the test's clinical utility and cost-effectiveness.

\section{Conclusion}

Prolonged TAT associated with sending out CSF EV PCR to a reference laboratory has clinical impact and associated costs. Expediting TAT to same-day result could potentially reduce unnecessary hospital LOS and intravenous antimicrobial therapy and thereby be cost saving.

Funding information This work was supported by the Fonds de recherche Québec santé (to J.P. and C.P.Y.). The funder had no role in the study design, data collection and analysis, decision to publish, or preparation of the manuscript. The other authors received no external funding. The authors have no financial disclosures to declare. 


\section{Compliance with ethical standards}

This study was approved by the McGill University Health Centre research ethics board (2018-4190).

Conflict of interest J.P. has received consulting/honoraria fees or research grant funding outside of the current work from the following: AbbVie; BD Diagnostics; Cepheid; MedImmune; Hoffmann-La Roche; Jannsen Pharmaceutical; Seegene. C.C. has received honoraria fees outside of the current work from Roche Diagnostics Canada. C.P.Y. has received in-kind support for investigator-initiated research projects from bioMérieux and BD Diagnostics in the last 3 years. The remaining authors declare no conflicts of interest.

\section{References}

1. Rotbart HA (1995) Enteroviral infections of the central nervous system. Clin Infect Dis 20(4):971-981

2. Rudolph H, Schroten H, Tenenbaum T (2016) Enterovirus infections of the central nervous system in children: an update. Pediatr Infect Dis J 35(5):567-569

3. Wallace SS, Lopez MA, Caviness AC (2017) Impact of enterovirus testing on resource use in febrile young infants: a systematic review. Hosp Pediatr 7(2):96-102

4. King RL, Lorch SA, Cohen DM, Hodinka RL, Cohn KA, Shah SS (2007) Routine cerebrospinal fluid enterovirus polymerase chain reaction testing reduces hospitalization and antibiotic use for infants 90 days of age or younger. Pediatrics 120(3):489-496

5. Ramers C, Billman G, Hartin M, Ho S, Sawyer MH (2000) Impact of a diagnostic cerebrospinal fluid enterovirus polymerase chain reaction test on patient management. JAMA 283(20):2680-2685

6. Archimbaud C, Chambon M, Bailly JL, Petit I, Henquell C, Mirand A, Aublet-Cuvelier B, Ughetto S, Beytout J, Clavelou P, Labbe A, Philippe P, Schmidt J, Regagnon C, Traore O, Peigue-Lafeuille H (2009) Impact of rapid enterovirus molecular diagnosis on the management of infants, children, and adults with aseptic meningitis. J Med Virol 81(1):42-48

7. Leber AL, Everhart K, Balada-Llasat JM, Cullison J, Daly J, Holt S, Lephart P, Salimnia H, Schreckenberger PC, DesJarlais S, Reed SL, Chapin KC, LeBlanc L, Johnson JK, Soliven NL, Carroll KC, Miller JA, Dien Bard J, Mestas J, Bankowski M, Enomoto T, Hemmert AC, Bourzac KM (2016) Multicenter evaluation of BioFire FilmArray meningitis/encephalitis panel for detection of Bacteria, viruses, and yeast in cerebrospinal fluid specimens. J Clin Microbiol 54(9):2251-2261

8. Giulieri SG, Chapuis-Taillard C, Manuel O, Hugli O, Pinget C, Wasserfallen JB, Sahli R, Jaton K, Marchetti O, Meylan P (2015) Rapid detection of enterovirus in cerebrospinal fluid by a fullyautomated PCR assay is associated with improved management of aseptic meningitis in adult patients. J Clin Virol 62:58-62

9. Panuganti SKN, S. (2018) Acute bacterial meningitis beyond the neonatal period. In: Long SSP, C. G.; Fischer, M. (ed) Principles and Practice of Pediatric Infectious Diseases. Elsevier, pp 278287.e272

10. Thomson J, Sucharew H, Cruz AT, Nigrovic LE, Freedman SB, Garro AC, Balamuth F, Mistry RD, Arms JL, Ishimine PT, Kulik DM, Neuman MI, Shah SS, Pediatric Emergency Medicine Collaborative Research Committee HSVSG (2018) Cerebrospinal fluid reference values for young infants undergoing lumbar puncture. Pediatrics 141(3):e20173405

11. Kestenbaum LA, Ebberson J, Zorc JJ, Hodinka RL, Shah SS (2010) Defining cerebrospinal fluid white blood cell count reference values in neonates and young infants. Pediatrics 125(2):257-264

12. Semret M, Schiller I, Jardin BA, Frenette C, Loo VG, Papenburg J, McNeil SA, Dendukuri N (2017) Multiplex respiratory virus testing for antimicrobial stewardship: a prospective assessment of antimicrobial use and clinical outcomes among hospitalized adults. J Infect Dis 216(8):936-944

13. AlGhounaim M, Xiao Y, Caya C, Papenburg J (2017) Diagnostic yield and clinical impact of routine cell culture for respiratory viruses among children with a negative multiplex RT-PCR result. J Clin Virol 94:107-109

14. Biondi EA, McCulloh R, Staggs VS, Garber M, Hall M, Arana J, Barsotti B, Natt BC, Schroeder AR, Schroeder L, Wylie T, Ralston SL (2019) Reducing variability in the infant sepsis evaluation (REVISE): a National Quality Initiative. Pediatrics 144(3)

15. McCulloh RJ, Fouquet SD, Herigon J, Biondi EA, Kennedy B, Kerns E, DePorre A, Markham JL, Chan YR, Nelson K, Newland JG (2018) Development and implementation of a mobile devicebased pediatric electronic decision support tool as part of a national practice standardization project. J Am Med Inform Assoc 25(9): $1175-1182$

16. Byington CL, Reynolds CC, Korgenski K, Sheng X, Valentine KJ, Nelson RE, Daly JA, Osguthorpe RJ, James B, Savitz L, Pavia AT, Clark EB (2012) Costs and infant outcomes after implementation of a care process model for febrile infants. Pediatrics 130(1):e16-e24

17. Timurkan MO, Aydin H, Sait A (2019) Identification and molecular characterisation of bovine parainfluenza virus-3 and bovine respiratory syncytial virus - first report from Turkey. J 63(2):167-173

18. Otto MP, Toyer AL, Poggi C, Janvier F (2019) Influenza B falsepositive results by rapid molecular tests AlereTM $\mathrm{i}$ influenza $\mathrm{a} \& \mathrm{~B} 2$ in France. Diagn Microbiol Infect Dis 94(4):342-343

19. Robinson CC, Willis M, Meagher A, Gieseker KE, Rotbart H, Glode MP (2002) Impact of rapid polymerase chain reaction results on management of pediatric patients with enteroviral meningitis. Pediatr Infect Dis J 21(4):283-286

20. Aronson PL, Lyons TW, Cruz AT, Freedman SB, Okada PJ, Fleming AH, Arms JL, Thompson AD, Schmidt SM, Louie J, Alfonzo MJ, Monuteaux MC, Nigrovic LE, Pediatric Emergency Medicine Clinical Research Network Herpes Simplex Virus Study G (2017) Impact of enteroviral polymerase chain reaction testing on length of stay for infants 60 days old or younger. J Pediatr 189(169174):e162

21. Nigrovic LE, Chiang VW (2000) Cost analysis of enteroviral polymerase chain reaction in infants with fever and cerebrospinal fluid pleocytosis. Arch Pediatr Adolesc Med 154(8):817-821

22. Basmaci R, Mariani P, Delacroix G, Azib S, Faye A, Taha MK, Bingen E, Bonacorsi S, Romero JR, Rotbart HA, Nyquist AC, Nolte FS (2011) Enteroviral meningitis does not exclude concurrent bacterial meningitis. J Clin Microbiol 49(9):3442-3443

23. Asner SA, Petrich A, Hamid JS, Mertz D, Richardson SE, Smieja M (2014) Clinical severity of rhinovirus/enterovirus compared to other respiratory viruses in children. Influenza Other Respir Viruses 8(4):436- 442

Publisher's note Springer Nature remains neutral with regard to jurisdictional claims in published maps and institutional affiliations. 